\title{
Schwer kranken Kindern ohne Diabetes nützt Insulintherapie nichts
}

Fragestellung: Profitieren schwer kranke Kinder von einer strengen Kontrolle erhöhter Blutglukosewerte?

\begin{abstract}
Hintergrund: Bei schweren Erkrankungen kommt es häufig auch bei Nichtdiabetikern zu Blutzuckeranstiegen. Diese gehen Beobachtungsstudien zufolge mit einer ungünstigen Prognose einher. Zwei kontrollierte randomisierte Studien bei Erwachsenen haben gezeigt, dass bei einer strengen Einstellung der Glukosewerte auf ca. 80 - $110 \mathrm{mg} / \mathrm{dl}$ die Mortalität höher ist als bei Werten unter $180 \mathrm{mg} / \mathrm{dl}$. Vergleichbare Studien bei Kindern sind widersprüchlich.
\end{abstract}

Patienten und Methoden: In eine multizentrische Studie wurden Kinder und Jungendliche im Alter von zwei Wochen bis 17 Jahren aufgenommen, die schwer erkrankt waren und wegen Hypotonie vasoaktive Substanzen benötigten oder beatmet werden mussten. Sie hatten keinen Diabetes und keinen kardialen Eingriff, aber Blutglukosewerte über $130 \mathrm{mg} / \mathrm{dl}$. Mittels einer kontinuierlichen intravenösen Insulininfusion mit fortlaufender Blutzuckerkontrolle wurden nach Zufallskriterien Werte zwischen 80 bis $110 \mathrm{mg} / \mathrm{dl}(4,4-6,1 \mathrm{mmol} / \mathrm{l} ; \mathrm{n}=360)$ oder 150 bis $180(8,3-10,0 ; \mathrm{n}=353)$ angestrebt.

Ergebnisse: Das Safety Board empfahl den vorzeitigen Abbruch der Studie, weil ein positives Ergebnis unwahrscheinlich war

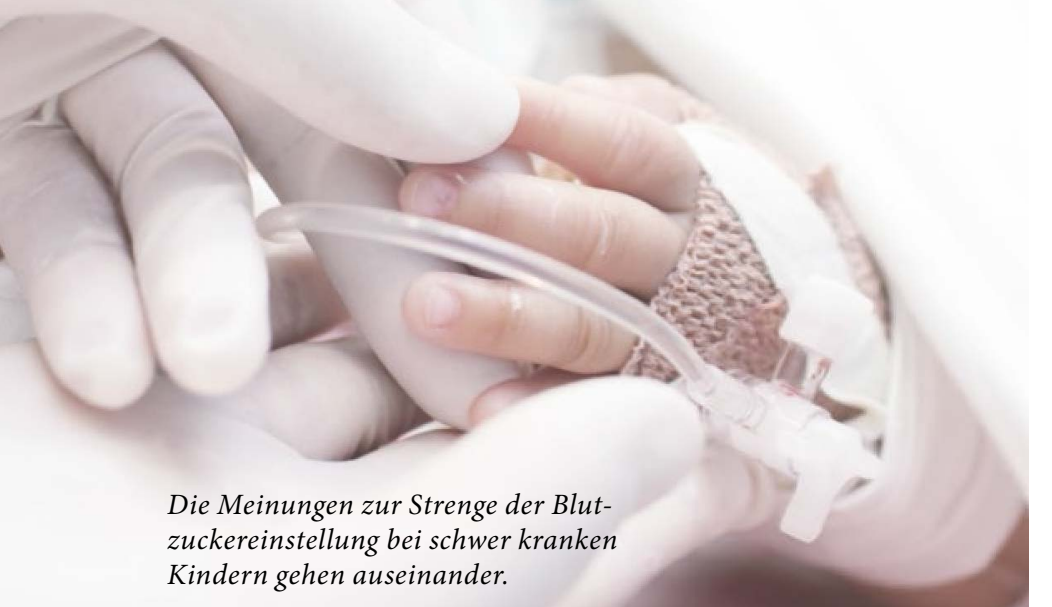

Die Meinungen zur Strenge der Blutuckereinstellung bei schwer kranken Kindern gehen auseinander. und sich Nachteile der intensiven Behandlung andeuteten. Bis zu diesem Zeitpunkt betrugen die mittleren Glukosewerte 109 bzw. $123 \mathrm{mg} / \mathrm{ml}$ unter intensiver bzw. wenig strenger Einstellung. Der primäre Endpunkt der Studie, die Zahl der Tage ohne Intensivstation bis zum 28 Krankheitstag war in beiden Gruppen mit 19,4 Tagen identisch.

Die Patienten mit niedrigen Zielwerten hatten signifikant häufiger Infektionen (3,4 vs. 1,1 \%) und Hypoglykämien unter $40 \mathrm{mg} / \mathrm{dl}$ (5,2 versus 2,0\%), während die Mortalität nach 28 Tagen höher lag, aber keine Signifikanz erreichte (13,5 versus 9,2 $\% ; \mathrm{p}=0,09)$.
Originalie

Agus M S D, Wypij D, Hirshberg E L et al. Tight Glycemic Control in Critically ill Children. N Engl J Med 2017;376:729-41
Schlussfolgerung: Eine strenge Blutzuckereinstellung von schwer kranken Kindern mit Hyperglykämie bringt keinen Nutzen, möglicherweise sogar Nachteile.

\section{- Kommentar von Prof. Dr. med. H. Holzgreve}

\section{Inkonsistente Studienergebnisse zu Insulin auf Intensiv}

Hyperglykämien im Verlauf schwerer Erkrankungen oder postoperativ bei Nichtdiabetikern signalisieren laut Beobachtungsstudien eine schlechte Prognose. Eine Insulintherapie auf der Intensivstation scheint da pathophysiologisch sinnvoll zu sein. Doch randomisierte kontrollierte Studien bei Patienten mit schweren Allgemeinerkrankungen oder nach kardialen Operationen und Verbrennungen bei Erwachsenen und Kindern geben kein einheitliches Bild. Bei einer Umfrage zu Studienbeginn vertraten Intensivmediziner gegenteilige Meinungen, teils lehnten Befürworter der Insulintherapie eine Mitwirkung an der Studie aus ethischen Gründen ab. In dieser Situation muss sich jeder ein eigenes Urteil bilden. Die Evidenz aus den bisherigen Studien spricht gegen eine strenge, aber für eine moderate Blutzuckersenkung.

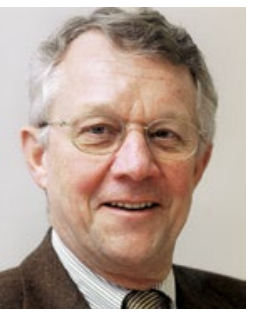

Prof. Dr. med. Heinrich Holzgreve

Internist, Kardiologische Praxis

Burgstr. 7, 80331 München

heinrich.holzgreve@t-online.de 